\title{
Competing Global and Local Completions in Visual Occlusion
}

\author{
R. J. van Lier, P. A. van der Helm, and E. L. J. Leeuwenberg \\ Nijmegen Institute for Cognition and Information, University of Nijmegen
}

\begin{abstract}
In visual occlusion 2 amodal-completion tendencies occur frequently. One tendency leads toward the simplest completed shape (a global completion) and the other to a shape for which the completion itself is as simple as possible (a local completion). Two experimental paradigms were used to test the strengths of these completion tendencies: a drawing task and a simultaneous matching task. The experimental results support the notion that the preference for either a global or a local completion is the consequence of a competition between interpretations. Finally, the authors discuss how the preference for a completion can be predicted by a model that is based on a quantification of both global and local aspects.
\end{abstract}

Objects might be positioned such that one object partly occludes another object. In many cases it appears that the visible part of the occluded shape is perceptually completed. This phenomenon has been referred to as amodal completion because the subjective presence of the occluded part of an object emerges without any modal characteristics (cf. Gerbino \& Salmaso, 1987; Kanizsa, 1985; Kanizsa \& Gerbino, 1982). During past decades several studies on visual occlusion and amodal completion have been done by various researchers (cf. Boselie, 1988; Boselie \& Wouterlood, 1989; Buffart, Leeuwenberg, \& Restle, 1981; Chapanis \& McCleary, 1953; Dinnerstein \& Wertheimer, 1957; Gerbino \& Salmaso, 1987; Kanizsa \& Gerbino, 1982; Kellman \& Shipley, 1991; Sekuler \& Palmer, 1992; van Lier, van der Helm, \& Leeuwenberg, 1994; Wouterlood \& Boselie, 1992). In the literature two types of amodal completion are frequently described: According to a global completion, the completed shape is as simple as possible, and according to a local completion, the completion itself is as simple as possible. In Figure 1 two different completions of a pattern are given. Figure 1A shows the global shape, that is, the shape resulting from the global completion. Figure 1B shows the local shape, that is, the shape resulting from the local completion.

In the remainder of this article we call patterns like that in Figure 1 GLD patterns (global-local-divergent) because global and local completions of such a pattern yield different shapes. This contrasts with GLC patterns (global-localconvergent) for which global and local completions yield

R. J. van Lier, P. A. van der Helm, and E. L. J. Leeuwenberg, Nijmegen Institute for Cognition and Information, University of Nijmegen, Nijmegen, The Netherlands.

We gratefully acknowledge the Netherlands Organization for Scientific Research (NWO) for funding this project. This research was conducted while R. J. van Lier was supported by Grant 560 262-039 from the Foundation for Behavioral Sciences. We wish to thank J. Lappin, W. Gerbino, and S. Palmer for their valuable comments on an earlier version of this article.

Correspondence concerning this article should be addressed to R. J. van Lier, Nijmegen Institute for Cognition and Information, University of Nijmegen, P.O. Box 9104, $6500 \mathrm{HE}$, Nijmegen, The Netherlands. the same shape (see Figure 2). (Of course, still other, more complex completions are possible, like Shape B in Figure 2, but we do not consider them because they are hardly perceptually relevant.)

It is important to realize that the exact shape that is called global or local depends on the employed notion of global and local simplicity. Consequently, the GLC-GLD classification made above also depends on the employed notion of simplicity.

Theories that emphasize local simplicity bear heavily on the role of the gestalt principle of good continuation in pattern completion. In this article we assume that, according to this principle, a local completion is constituted by a linear continuation of the visible contours of the background shape behind the occluding shape, as shown in Figures 1B and 2A. Other operationalizations of the good continuation principle are also possible. Kellman and Shipley (1991) argued that a completion will be such that points of occlusion are connected by the simplest continuous function. However, the generalizability of this assumption has been criticized by Boselie and Wouterlood (1992), who mentioned several limitations of the hypothesis of Kellman and Shipley. Nevertheless, Wouterlood and Boselie (1992) presented a local completion theory that is based on the good continuation principle as well. The application of this principle in combination with predefined junction types, which might occur between two surfaces, enables them to predict when occlusion is experienced. Whenever an occlusion occurs, the visible part of the background shape is completed by means of linear good continuation. However, up until now their theory is restricted to configurations of two completely irregular surfaces. As Wouterlood and Boselie themselves already indicated, this restriction implies that typical global characteristics of patterns are ignored.

In contrast with local theories, global theories take into account all regularities within a pattern. Early gestalt psychologists (Köhler, 1947; Wertheimer, 1923) discussed already the relevance of regularities for visual perception. The gestalt law of Prägnanz (Koffka, 1935) embodies the concept that perception tends to result in overall simplicity. This gestalt law can be regarded as the predecessor of the global minimum principle. The global minimum principle 


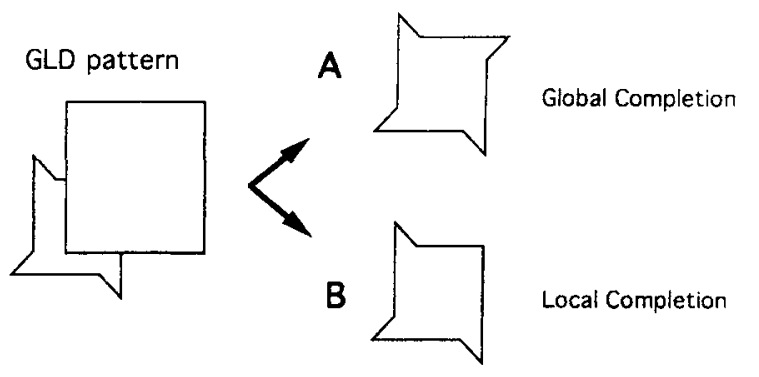

Figure 1. Two possible occlusion interpretations of the same pattern. Interpretation A represents the simplest global completion and Interpretation B the simplest local completion. Because global and local completions of the pattern diverge into different shapes, the pattern is referred to as a GLD pattern (global-localdivergent).

(Hochberg \& McAlister, 1953) conjoined with a descriptive system of visual patterns (cf. Hochberg \& McAlister, 1953; Leeuwenberg, 1969, 1971; Restle, 1982) results in clear predictions of the preferred pattern interpretations. The encoding of patterns may proceed in various ways. For the moment, we assume the application of the minimum principle in visual occlusion to be such that the simplest shape, according to a specific coding scheme, is predicted to be preferred. Following the coding procedure within structural information theory (SIT; van der Helm \& Leeuwenberg, 1991), the shapes in Figures 1A and 2A indeed appear to be the simplest for the given patterns. (The coding rules and the encoding procedure are briefly discussed in the Appendix.) However, the global minimum principle has been criticized too by several researchers who demonstrated that the preferred interpretation does not always coincide with the interpretation with the simplest shape (cf. Boselie, 1988; Kanizsa, 1985; Rock, 1983). The pattern given in Figure 3 was brought in by Kanizsa (1985). Interpretation $A$ in Figure 3 is supposed to agree with the global minimum principle, yet Interpretation B generally is preferred to A.

The majority of arguments of proponents or opponents of a given theory in this global-local discussion on visual completion comprises phenomenal examples and counterexamples for one or the other theory. Apparently, in some cases the perceptual process results in an interpretation that can be classified as a global completion, whereas in other cases a local completion is more prevalent. Given this fact, a theory that is strictly based on either global simplicity or local simplicity cannot hold. In our view a theory of visual completion has to incorporate both global and local aspects of pattern interpretations. In this article we discuss two questions: (a) Can the preferred completion be conceived as the result of a competition between global and local completions? (b) How can global and local aspects be accounted for in the selection of an interpretation? Before we deal with these questions, we first present an introductory experiment that demonstrates the relevance of both global and local completions.

\section{Experiment 1: Both Global and Local Completions Are Relevant}

\section{Method}

Participants. Twenty-one undergraduate students served as participants. All participants received course credit.

Stimuli. The stimulus set consisted of the GLD patterns given in Figure 4. The construction of these patterns started with one of six symmetrical shapes, containing mirror symmetry (with three or four axes of symmetry) or rotation symmetry. Each symmetrical shape was partly occluded by a rectangle, such that they constituted a GLD pattern. The global completion of each GLD pattern resulted in the symmetrical shape, whereas the local completion was reached by linear good continuation of the visible part of the occluded shapes. Two stimulus subsets were designed. Within Stimulus Subset 1 a relatively small part of the symmetrical shape was occluded, whereas within Stimulus Subset 2 a relatively large part of the symmetrical shape was occluded. As a consequence of this procedure, the global shape was the same in both stimulus subsets, whereas the local shape varied between the stimulus subsets. For reasons of comparability with Experiment 2B, the relative orientation of each shape with respect to the occluding rectangle was such that the rectangle had the same (upright) position for all patterns. Pattern height was between $3 \mathrm{~cm}$ and $5 \mathrm{~cm}$.

The main goal of the manipulation described above was the creation of a set of patterns in which the preference for a specific global completion could be tested for two patterns with different local completions.

Procedure. The patterns were presented in random order on pieces of paper. The orientation of each pattern was as given in Figure 4. The participants were told that every drawing represented two surfaces: a rectangle and a shape behind the rectangle. The participants were asked to draw the contours of the occluded part of the perceived background shape for each pattern.

\section{Results}

Within Stimulus Subset 1, global completions were preferred in $81 \%$ of all cases and local completions in $13 \%$ of all cases. Within Stimulus Subset 2, global completions were preferred in $28 \%$ of all cases and local completions in $64 \%$ of all cases. The percentages of global and local

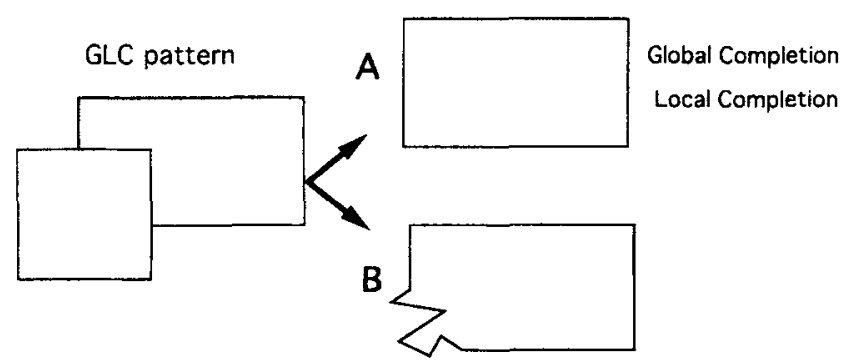

Figure 2. Two possible occlusion interpretations of the same pattern. Interpretation $A$ represents both the simplest global and the simplest local completion. Because global and local completions converge to the same shape, the pattern is referred to as a GLC pattern (global-local-convergent). Other, more complex, interpretations, like Interpretation $\mathbf{B}$, are also possible but are hardly perceptually relevant. 


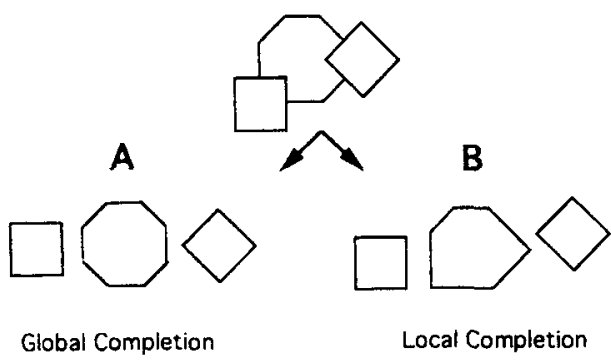

Figure 3. A GLD pattern (global-local-divergent) adopted from Kanizsa (1985). Interpretation A represents the global completion and Interpretation B the local completion. The local completion appears to be more prevalent than the global completion.

completions do not sum up to $100 \%$ exactly, because some completions did not fit the present definition of global or local completion. In Table 1 the proportion of preference for the global completion is presented for each pattern. This proportion is calculated by the number of participants $(N)$ who preferred (Pref) the global completion divided by the number of participants who preferred either the global or the local completion: $\operatorname{Pref}_{\text {global local }}=N_{\text {global }} /\left(N_{\text {global }}+N_{\text {local }}\right)$.

\section{Discussion}

The data clearly illustrate that both global and local completions are relevant in pattern completion. The manipulation for generating both stimulus subsets may have affected several factors that caused the overall shift in preference from global completions in Stimulus Subset 1 to local completions in Stimulus Subset 2. Later in this article we deal with the question of which factors may be responsible for the preference for either a global or a local completion. For the moment we maintain our statement that any theory on pattern completion that emphasizes either global simplicity or local simplicity fails. We now turn to the first question.

\section{Question 1: Can the Preferred Completion Be Conceived as the Result of a Competition?}

The competition between alternative interpretations is not an obvious fact, at least according to various perceptual theories. Perceptual models that aim at a minimization of process, such as a hill-climbing procedure (Attneave, 1982), typically result in only one interpretation. Also, theories in which local cues such as junction types (cf. Barrow \& Tenenbaum, 1981; Wouterlood \& Boselie, 1992) or nonaccidental properties (Biederman, 1987) play a dominant role lead in principle to just one interpretation. In some cases conflicting cues may lead to a predicted ambiguity (cf. Wouterlood \& Boselie, 1992), but this does not imply the assumption of a competition between different interpretations for nonambiguous patterns. In contrast with this, perceptual theories that use a selection criterion for the perceived interpretation use the idea of more possible candidates. Both the likelihood principle (cf. Von Helm- holtz, 1867/1962) and the minimum principle (Hochberg \& McAlister, 1953; see also Hatfield \& Epstein, 1985) can be conceived as such. Already Herbart (1850) proposed that the degree in which one interpretation prevails over another is the result of a competition between different interpretations. Mens and Leeuwenberg (1988) resumed two basic ideas in Herbart's theorizing: (a) the conscious percept is but one out of many subliminal candidates and (b) the dominant percept may vary in strength, depending on the attractiveness of the competing interpretations. In addition, Mens and Leeuwenberg demonstrated the hidden presence of alternative interpretations for certain visual patterns.

In line with the latter ideas, the competition between global and local completions may gain support by a possible interdependency of the strengths of both types of completions. In the following experiments this interdependency is tested by means of the simultaneous matching task (Gerbino \& Salmaso, 1987).

\section{Simultaneous Matching Task}

We first review parts of the study by Gerbino and Salmaso (1987). Consider Figure 5. In one of their experiments
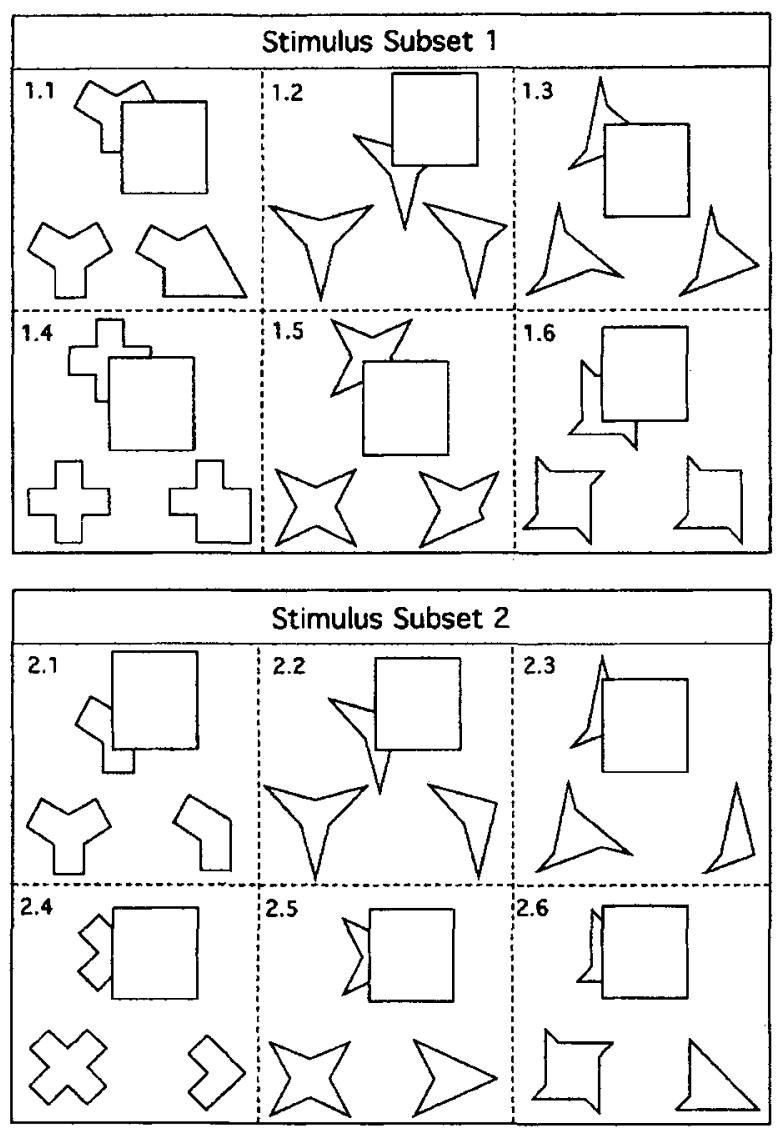

Figure 4. The patterns used in Experiment 1. All patterns are GLD patterns (global-local-divergent). For each pattern the global completion is drawn on the left and the local completion on the right. 
Table 1

Experiment 1 (Drawing Task): Relative Preference for Each Global-Local-Divergent Pattern

\begin{tabular}{cc}
\hline Pattern & Pref $_{\text {globallocal }}$ \\
\hline 1.1 & 0.95 \\
1.2 & 0.86 \\
1.3 & 0.50 \\
1.4 & 0.95 \\
1.5 & 1.00 \\
1.6 & 0.85 \\
2.1 & 0.35 \\
2.2 & 0.33 \\
2.3 & 0.24 \\
2.4 & 0.38 \\
2.5 & 0.30 \\
2.6 & 0.22 \\
\hline
\end{tabular}

Note. For each pattern the preference (Pref) for the global completion relative to the local completion is given (Pref globallocal $_{\text {) }}$. Pref $_{\text {globallocal }}=$ number of global completions/(number of global completions + number of local completions).

patterns like A and B were presented simultaneously with shapes such as $C$ or D. Participants were asked to indicate as quickly as possible whether the simultaneously presented single shape was present in the pattern. It appeared that positive responses on the presence of Shape $C$ in Pattern $A$ required about the same reaction times as did the positive responses on the presence of the same shape in Pattern $B$. Furthermore, positive responses on the presence of Shape D in Pattern B required significantly longer reaction times than did positive responses on the presence of Shape $C$ in Pattern B.

Gerbino and Salmaso (1987) concluded that amodally completed shapes are functionally equivalent to complete shapes. Gerbino and Salmaso further remarked that their experiments were not designed to test whether completion tends to be global or local. Indeed, their stimulus set consisted of GLC patterns, so that both global and local completion strategies converged to the same completed shape. In case of GLD patterns, however, the response times on global and local completions may be influenced by their relative strengths.

\section{Experiment $2 A$ (Pilot Experiment)}

We adopted only part of the experimental procedure of Gerbino and Salmaso (1987). For this reason we first present a pilot experiment to examine whether the present procedure leads to the same results on GLC patterns as Gerbino and Salmaso found. We focus on the question of whether matching a shape with a partly occluded shape evokes the same response times as matching it with a completely visible shape.

\section{Method}

Participants. Twenty-two undergraduates participated in the experiment. All participants received course credit.

Stimuli and procedure. Like Gerbino and Salmaso (1987), we used line drawings. The complete stimulus set was based on six patterns and three target shapes (see Figure 6). In the following discussion we refer to this stimulus set as the GLC set. Each pattern consisted of a rectangle with a second shape positioned either behind or in front of that rectangle, which represented the occlusion condition and the no-occlusion condition, respectively. (Evidently the distinction occlusion-no-occlusion refers to the position of the nonrectangular shape.) In a stimulus presentation each pattern was paired with a target shape. This target shape either could be matched with the (nonrectangular) shape within the pattern or could not be matched with that shape. In this way a small set of 12 stimuli was constructed, for which the amounts of correct yes and no answers were balanced. In Figure 7 a schematic presentation of the events in a trial is shown.

At time $T=0$ a rectangle $\left(1.5^{\circ}\right.$ wide) was presented in the upper half of a monitor screen. After $2 \mathrm{~s}$ an additional shape, Shape $\mathrm{A}$, appeared either in front of the rectangle (no-occlusion condition, see Figure $7 \mathrm{~A}$ ) or partly behind the rectangle (occlusion condition, see Figure 7B). At the same time, another shape, Shape B, was presented in the lower half of the screen. The total visual angle was about $6^{\circ}$.

The task of the participant was to verify whether Shape B could be matched with Shape A. If Shape A partly covered the rectangle (Figure 7A), the participant had to respond whether Shape $A$ and Shape $B$ were the same. If Shape $A$ was partly covered by the rectangle (Figure 7B), the participant had to respond whether Shape A could be the same as Shape B. Responses were given by pressing one of two buttons (yes or no). Participants were asked to respond as quickly as possible. The response time was measured, being the time from the appearance of Shapes A and B until a button was pressed. The patterns remained on the screen until a response was given. Before running the experiment, the participants were trained on a set of stimuli different from the stimuli used in the experiment. During the training period, participants were given feedback on their responses. During the experiment, no feedback was given. The order of presentation of stimuli was randomized for each participant.

\section{Results and Discussion}

The results are shown in Table 2. For each combination the mean response time for the correct answers, the standard error of the response times, and the percentage of incorrect answers (relative to the amount of stimuli per cell) are given. An analysis of variance (ANOVA) for repeated mea-
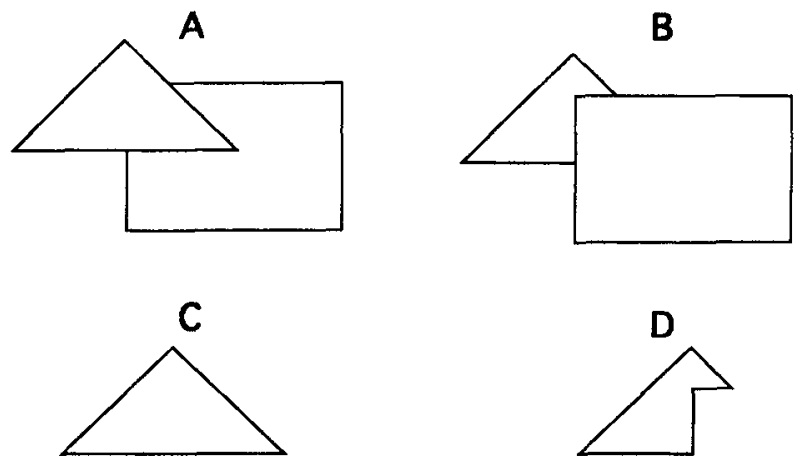

Figure 5. According to Gerbino and Salmaso (1987) the recognition of Shape $C$ in Pattern A occurs within the same time span as the recognition of Shape C in Pattern B. Moreover, in Pattern B, Shape $C$ is recognized more rapidly than Shape D. 


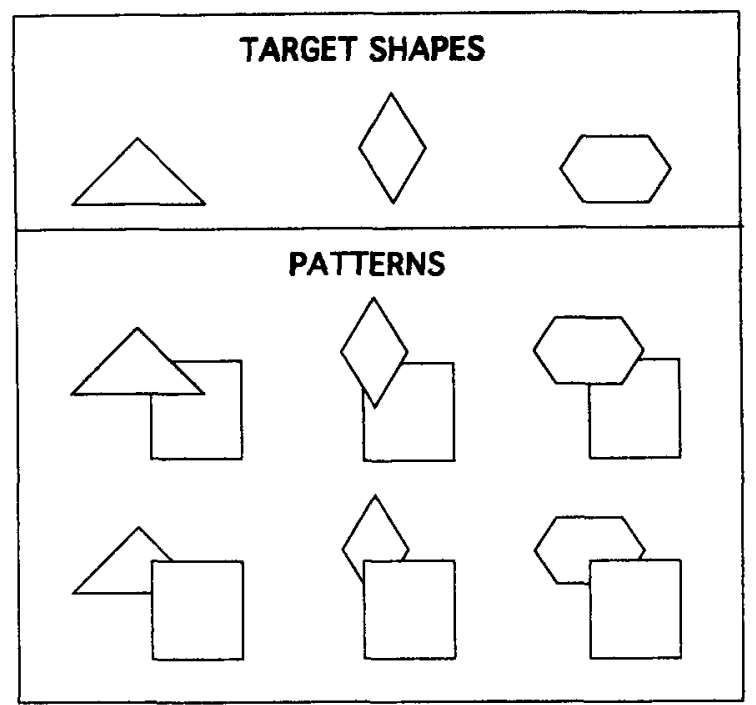

Figure 6. Target shapes and patterns as used in Experiment 2A.

surements on these data has been performed, revealing no main effect on occlusion, $F(1,21)=0.55, p>.10$. That is, the response time on matching a shape with a completely visible shape was not significantly different from the response time on matching it with a partly occluded shape. In concordance with the results of Gerbino and Salmaso (1987) there was a significant main effect on match, $F(1$, 21) $=8.41, p<.01$. The Occlusion $\times$ Match interaction was not significant, $F(1,21)=0.38, p>.10$. Finally, a $t$ test was performed on the responses for stimuli in which a match was possible (two upper rows in Table 2), which revealed no significant effect for occlusion $t(21)=0.89$, $p>.10$.

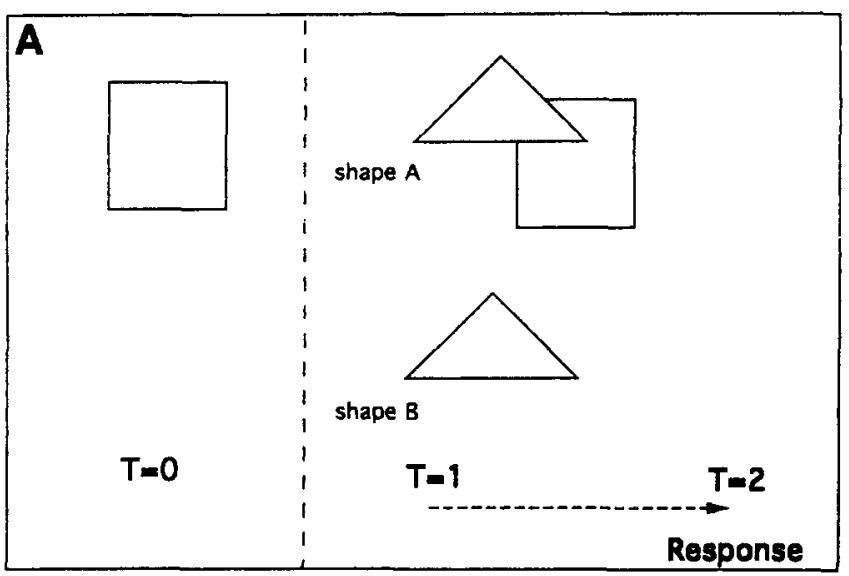

Figure 7. A schematic layout of the events in a stimulus presentation of Experiment 2. At $T=0$, a rectangle was presented. After $2 \mathrm{~s}(T=1)$ a shape (Shape A) appeared either in front of the rectangle (see Figure 7A) or partly behind the rectangle (see Figure 7B). Also at $T=1$, a second shape (Shape B) appeared. The participant had to verify whether Shape B could be matched with Shape A. Response times were measured, being the time from $T=1$ until a button (yes or no) was pressed (at $T=2)$.

The results on the present experimental procedure confirm Gerbino and Salmaso's (1987) findings for GLC patterns. Therefore, we can now turn to the application of this procedure to GLD patterns.

\section{Experiment $2 B$}

\section{Method}

Participants. Thirty-one undergraduates participated in the experiment. All participants received course credit.

Stimuli and procedure. The same patterns as in Experiment 1, having the same orientations, were used. Each of the 12 GLD patterns of Experiment 1 led to 8 stimuli in the present experiment, so that in total there were 96 stimuli. An example is given in Figure 8.

Every pattern-target combination in Figure 8 represents a stimulus as used in the experiment. Pattern D was added to the set of combinations to balance the amounts of correct yes and no answers. In the following discussion we refer to this stimulus set as the GLD set. The stimulus presentation procedure was the same as in Experiment 2A.

\section{Results and Discussion}

In Table 3, overall data are presented. An ANOVA for repeated measurements on these data revealed a significant main effect on occlusion, $F(1,30)=40.3, p<.001$, a significant main effect on match, $F(1,30)=9.55, p<.01$, and an Occlusion $\times$ Match interaction, $F(1,30)=63.8$, $p<.001$.

To test the differences between the GLC set and the GLD set, an ANOVA with set (GLC vs. GLD) as a betweensubjects variable was performed on the data of both Experiments $2 \mathrm{~A}$ and $2 \mathrm{~B}$. There was a significant main effect on

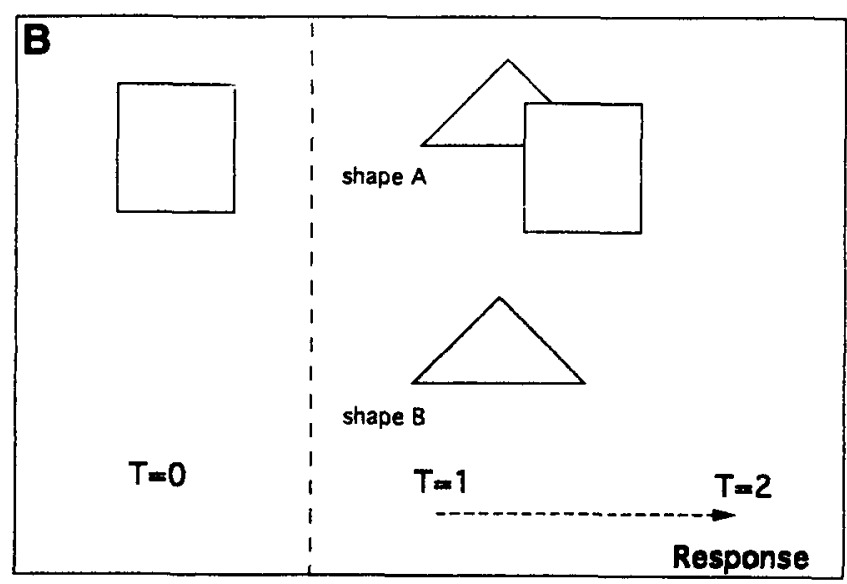


Table 2

Experiment 2a (Matching Task): Overall Results on Global-Local-Convergent Patterns

\begin{tabular}{lccc}
\hline \multicolumn{1}{c}{ Combination } & RT (ms) & SE (ms) & ER (\%) \\
\hline Match/occlusion & 736.5 & 40.2 & 1.5 \\
Match/no occlusion & 712.5 & 27.1 & 1.5 \\
No match/occlusion & 799.2 & 37.5 & 1.5 \\
No match/no occlusion & 799.3 & 44.2 & 3.0 \\
\hline
\end{tabular}

Note. $\mathrm{RT}=$ response time; $\mathrm{SE}=$ standard error; $\mathrm{ER}=$ error rate.

set, $F(1,51)=49.93, p<.001$. There was no Match $\times$ Set interaction, $F(1,51)=0.05, p>.10$, and a significant Occlusion $\times$ Set interaction, $F(1,51)=18.17, p<.001$. The threefold Occlusion $\times$ Match $\times$ Set interaction was also significant, $F(1,51)=27.5, p<.001$.

The overall high response times on the GLD set, in comparison with those on the GLC set, can be explained by the fact that the shapes within the GLD set are more complex than those within the GLC set. Most important, however, is that, in case of a possible match, the GLC set yielded no significant difference between the occlusion condition and the no-occlusion condition, whereas the GLD set yielded significantly longer response times in the occlusion condition in comparison with the no-occlusion condition. In Figure 9 the response times on possible matches are depicted for both the GLC set and the GLD set.

We now focus on the responses on possible matches in the GLD set. The data are split up with respect to stimulus subset (see Table 4) and type of shape (global vs. local). In Table 4 the mean response times are presented. Per participant, the response on a possible match in the occlusion condition and the response on a possible match in the no-occlusion condition, involving the same shape, were considered only if both responses were correct. This excludes $6 \%$ of all responses. In all cases the mean response times in the occlusion condition were higher than the mean response times in the no-occlusion condition. We performed $t$ tests on these differences, and they appeared to be highly significant $(p<.001$, see also Table 4$)$. For each shape representing a global or a local completion of a GLD pattern the difference in response times (DRT) between the noocclusion condition and the occlusion condition was calculated within participants (see also Figure 10). An ANOVA for repeated measurements performed on these values revealed no significant main effect on stimulus subset, $F(1$, $30)=1.67, p>.10$, a significant main effect on the type of shape (global vs. local), $F(1,30)=8.02, p<.01$, and a significant Stimulus Subset $\times$ Type of Shape interaction, $F(1,30)=20.74, p<.001$.

On the whole, the DRT values for the global shapes were the lowest for the patterns of Stimulus Subset 1, whereas the DRT values for the local shapes were the lowest for the patterns of Stimulus Subset 2. To relate the results of Experiment 1 with the results of Experiment 2B, we calculated for each pattern the mean DRT value of the global completion relative to the mean DRT values of both global and local completions: $\mathrm{DRT}_{\text {globallocal }}=$
$\mathrm{DRT}_{\text {global }} /\left(\mathrm{DRT}_{\text {global }}+\mathrm{DRT}_{\text {local }}\right.$ ) (see Table 5). Correlating the proportion of preference, Pref globallocal $_{\text {, of Experiment } 1}$ and the proportion $\mathrm{DRT}_{\text {globallocal }}$ of Experiment $2 \mathrm{~B}$, yields a Pearson's correlation coefficient of $-.93(p<.001$; see Figure 11). This indicates that, generally, the higher the preference for a completion is, the smaller the difference in response time between the occlusion condition and the no-occlusion condition will be.

The overall agreement between the results of both experiments indicates that the strength of the global completion depends on the strength of the local completion and vice versa. Note that from these experiments it cannot be deduced whether the different completions are concurrently present nor whether they are generated simultaneously or sequentially. The only argument here concerns the interdependency of the strengths of both types of completions. This interdependency supports the notion that the preference for either a global or a local completion is the result of a competition between those completions. We therefore pursue predictive models of pattern completion that account for that. This leads us to the second question.

\section{Question 2: How Can Global and Local Aspects Be Accounted For in the Selection of an Interpretation?}

Given the above results, it is clear that any model on pattern completion has to deal with both global and local completions. We first evaluate some factors inherent to two-dimensional patterns that might have influenced the preference for a specific completion. After this we focus on a relatively simple model for pattern completion.

\section{Some Factors in Pattern Completion}

\section{Pattern Orientation}

Effects of orientation on pattern completion have been reported by Boselie (1988). More specifically, Boselie reported a preference for local completions with a vertical axis of symmetry. The vertical axis appeared not to be a dominant factor with respect to the global completions. Also from the data of Experiment 1, it is clear that a vertical axis of symmetry is not a dominant factor. For example, Patterns 2.1, 2.2, 2.4, and 2.5 all had a vertical axis of

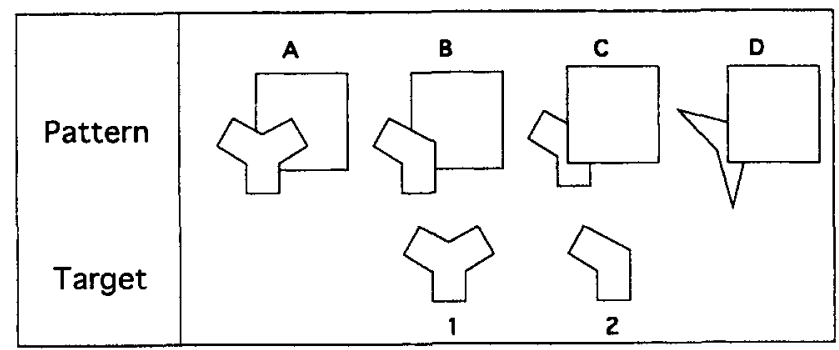

Figure 8. Each pattern-target combination represents a stimulus as used in Experiment $2 \mathrm{~B}$. 
Table 3

Experiment $2 b$ (Matching Task): Overall Results on Global-Local-Divergent Patterns

\begin{tabular}{lccc}
\hline \multicolumn{1}{c}{ Combination } & RT (ms) & SE (ms) & ER (\%) \\
\hline Match/occlusion & 1342.6 & 65.6 & 4.4 \\
Match/no occlusion & 1036.6 & 36.1 & 2.2 \\
No match/occlusion & 1237.8 & 54.3 & 5.4 \\
No match/no occlusion & 1276.2 & 51.7 & 9.6 \\
\hline
\end{tabular}

Note. $\mathrm{RT}=$ response time; $\mathrm{SE}=$ standard error; $\mathrm{ER}=$ error rate.

symmetry for the global shape only. Nevertheless, the local completion was preferred.

\section{Relative Orientation of Edges}

Kellman and Shipley (1991) and Shipley and Kellman (1992) reported an effect of the relative orientation of edges on perceived occlusion. According to their relatability criterion, edges are relatable when linear extension of occluded edges meet at obtuse angles or, as a limit case, at an angle of $90^{\circ}$ (referred to as marginally relatable edges). According to their hypothesis, edges that meet with an angle less than $90^{\circ}$ do not give rise to edge interpolation. Within Stimulus Subset 1, two patterns (1.4 and 1.5) have occluding edges that are marginally relatable, whereas within Stimulus Subset 2 , two patterns ( 2.2 and 2.4 ) have occluding edges that are marginally relatable and two patterns (2.1 and 2.3) with occluding edges that are relatable. If relative orientation of edges, expressed in terms of relatability, is taken as the only decisive variable with respect to the occurrence of a local completion, it is unclear why Patterns 2.5 and 2.6, having nonrelatable edges, lead to a local completion and why Patterns 1.4 and 1.5 , having marginally relatable edges, lead to a global completion.

\section{Familiarity}

According to Peterson and Hochberg (1989), interpretations that represent familiar figures are more likely to be preferred. Also Kellman and Shipley's (1991) notions on preferred completions in case of nonrelatable edges depend on familiarity. Others are critical on the role of familiarity in pattern completion (cf. Kanizsa, 1985; Kanizsa \& Gerbino, 1982). It is not always clear which predictions are made on the basis of this principle, as it lacks any quantification. In many cases the most regular shapes will be more familiar. Clearly, the overall responses on Stimulus Subset 2 contradict a tendency toward regularity. Familiarity obviously comprises more than regularity. Nevertheless, it cannot be the only crucial variable because many patterns, such as those in Wouterlood and Boselie (1992), yield strong preferences for unfamiliar completions.

\section{Context}

Somewhat related to the effects of familiarity are effects of context. The presence of other shapes in the visual field, with either spatial or temporal distance, could influence the preference for a certain interpretation (cf. Leeuwenberg, Mens, \& Calis, 1985; Rock, 1985). With respect to both Experiments 1 and 2B, the appearance of other regular shapes might have biased the result toward a higher preference for regular completions.

\section{Coincidence}

Rock (1983) argued that the perceptual system avoids coincidences within interpretations. In case of occlusion patterns, these coincidences may occur between the contours of the shapes. Rock, however, did not give a metric of coincidence, so it is difficult to make precise predictions on the basis of this principle as such. All of our experimental patterns were constructed such that both global and local completions did not yield coinciding contours. Because of the fact that coincidences can be regarded as unexplained regularities (Rock, 1983), we argued elsewhere that coincidence can be expressed in terms of a quantifiable complexity (van Lier et al., 1994). Also, according to that proposed quantification, both types of completions do not yield any coincidence. We conclude that the shift in preferences from global completions (Stimulus Subset 1) to local completions (Stimulus Subset 2) was not caused by a variation in coincidence.

\section{Local Configurations}

Somewhat related to the effects of coincidence are the effects of local configurations. For instance, whether a completion occurs and, if so, which completion occurs may depend on the type of junctions at the points of occlusion (cf. Ratoosh, 1949; Wouterlood \& Boselie, 1992). All of our experimental patterns were constructed such that $T$ junctions arose at all points of occlusion. Therefore, a variation

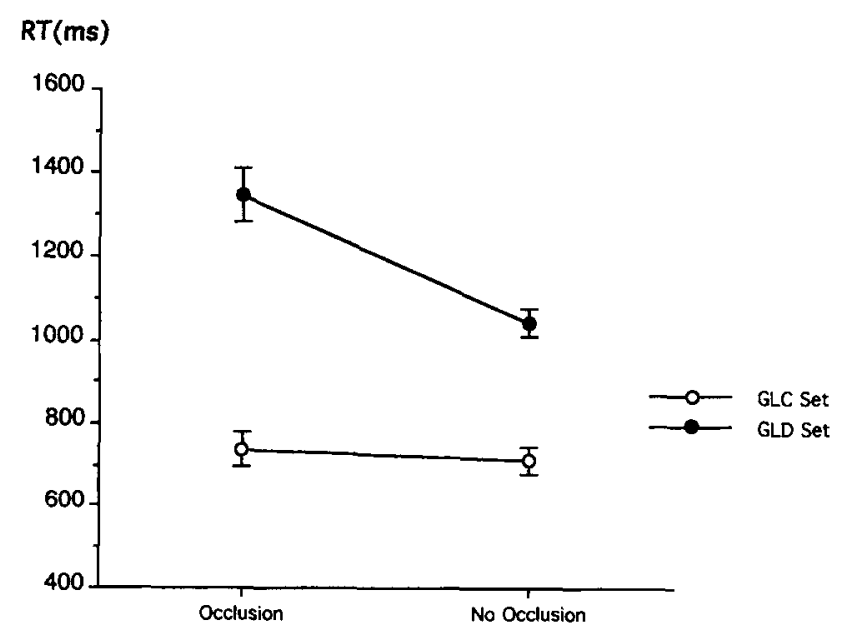

Figure 9. The response times (RT) on possible matches in the GLC set (global-local-convergent; Experiment 2A) and the GLD set (global-local-divergent; Experiment 2B). 
Table 4

Experiment $2 b$ (Matching Task): Mean Response Times on Possible Matches for Global-Local-Divergent Patterns

\begin{tabular}{lcccc}
\hline & \multicolumn{2}{c}{ Condition (ms) } & & \\
\cline { 2 - 3 } Shape & Occlusion & No occlusion & DRT & $t(30)$ \\
\hline \multicolumn{5}{c}{ Stimulus subset 1} \\
Global & 1157.2 & 969.3 & 187.9 & 4.67 \\
Local & 1621.3 & 1156.0 & 465.3 & 7.61 \\
\hline \multicolumn{5}{c}{ Stimulus subset 2} \\
Global & 1327.4 & 999.0 & 328.4 & 6.31 \\
Local & 1249.5 & 1038.0 & 211.5 & 7.71 \\
\hline
\end{tabular}

Note. In all cases the response times in the occlusion condition are higher than the response times in the no-occlusion condition. In all cases the $t$ tests are highly significant $(p<.001)$. DRT $=$ difference in response times.

in type of junctions at points of occlusion cannot have caused the shift in preferences.

\section{Simplicity of Shape}

The complexities of the global and local shapes in terms of structural information $(I)$ have been determined for each pattern. In the Appendix, the determination of $I$ is shown for Pattern 2.2. In Table 6, the $I$ values are given for each pattern of Figure 4. It appears that for all patterns the global shape is simpler than the local shape. So, if there is a dominant tendency toward the simplest shape, the global shape should be perceived for all patterns drawn in Figure 4. Clearly, the preferences on the patterns of Stimulus Subset 2 are in contradiction with this idea. One might argue that the local shapes of Stimulus Subset 2 are simpler than those of Stimulus Subset 1 and that this might have caused the shift to local completions in Stimulus Subset 2. For example, there are more local shapes in Stimulus Subset 2 that have an axis of symmetry. Consequently, the difference in complexity between global and local shapes is smaller for Stimulus Subset 2. However, the local shapes are still more complex than the global shapes and therefore will not be predicted on the basis of simplicity of shape. Moreover, given the information loads, the difference in complexity cannot be a factor with a strong predictive power because it is not clear what the absolute value of this difference has to be in order to reach the shift in completion. For instance, the difference in complexity (in terms of structural information) between the global and the local shapes for Pattern 1.4 and Pattern 2.3 is exactly the same for both patterns, yet for Pattern 1.4 the global completion is preferred, whereas for Pattern 2.3 the local completion is preferred.

None of the above factors alone can account for the preferences for either a global or a local completion. The list of discussed factors certainly is not exhaustive. Other factors, such as the lengths of edges at points of occlusion or the occluded area, might affect completion as well. An all-embracing model for the prediction of pattern completions has to deal with all possible factors. In the discussion below, however, we propose a relatively simple model on pattern completion, which is based only on the simplicity of the completed shape and the simplicity of the amodal part of that shape. Before that, we present a replication of Experiment 1 to control for two aspects that might have influenced the outcome of Experiments 1 and $2 \mathrm{~B}$, namely orientation and context. These aspects can be controlled without changing the patterns themselves.

\section{Experiment 3 (Replication of Experiment 1)}

\section{Method}

Participants. Two hundred eighty-eight undergraduate students participated voluntarily.

Stimuli. The same patterns as in Experiment 1 were used. To reduce effects of orientation, each pattern was presented in 24 orientations, with steps of $15^{\circ}$, starting with the orientation given in Figure 4 . In total there were $12 \times 24=288$ stimuli. The stimuli were drawn on pieces of paper.

Procedure. Each participant received only one stimulus. This was done to eliminate context effects of other stimuli. The instruction was the same as in Experiment 1. Again the participants were asked to draw the contours of the occluded part of the perceived background shape.

\section{Results}

Within Stimulus Subset 1, global completions were preferred in $69 \%$ of all cases and local completions in $22 \%$ of all cases. Within Stimulus Subset 2, global completions were preferred in $15 \%$ of all cases and local completions in $78 \%$ of all cases. In Table 7 the proportion of preference for the global completion is presented for each pattern. Note that, in comparison with Experiment 1, Pattern 1.1 shows a remarkable switch from a global to a local completion. For the other patterns the proportions generally are more extreme than in Experiment 1, especially for Patterns 2.1, 2.2, and 2.3 .

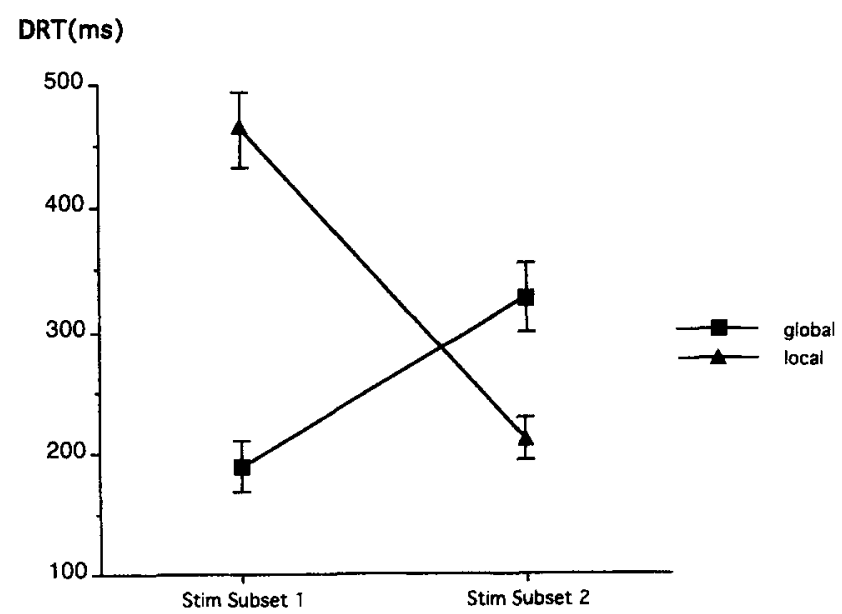

Figure 10. The mean difference in response times (DRT) values for possible matches in Experiment $2 B$ are plotted for both stimulus (Stim) subsets. 
Table 5

Experiment $2 B$ (Matching Task): Relative Difference in Response Time for Each Global-Local-Divergent Pattern

\begin{tabular}{cc}
\hline Pattern & DRT $_{\text {globallocal }}$ \\
\hline 1.1 & 0.23 \\
1.2 & 0.32 \\
1.3 & 0.67 \\
1.4 & 0.25 \\
1.5 & 0.19 \\
1.6 & 0.28 \\
2.1 & 0.63 \\
2.2 & 0.61 \\
2.3 & 0.64 \\
2.4 & 0.45 \\
2.5 & 0.63 \\
2.6 & 0.60 \\
\hline
\end{tabular}

Note. For each pattern the difference in response times (DRT) for the global completion relative to the local completion is given $\left(\right.$ DRT $\left._{\text {global local }}\right) . \mathrm{DRT}_{\text {globallocal }}=\mathrm{DRT}$ global completion/(DRT global completion + DRT local completion).

\section{A Simple Completion Model}

We start with the observation that in pattern completion there are two opposing tendencies. On the one hand, there is a tendency toward a global completion, which is characterized by an overall simplicity of the completed (background) shape but which may result in a rather complex amodal part of that shape. On the other hand, there is a tendency toward a local completion, which is characterized by a relatively simple amodal part of a shape but which may result in a more complex completed shape than under global completion. To deal with these opposing tendencies, we consider the perceptual complexity of an interpretation based on the complexity of both the completed shape and the amodal part of that shape. The complexity of the completed shape (global or local) has already been dealt with. To define the complexity of the amodal part of a shape we follow a proposal that has been made in van Lier et al. (1994). In that study we argued that the number of occluded, or "virtual," contour elements already provides a solid basis for an operationalization of the perceptual complexity of the amodal part. We refer to the complexity of the amodal part with $V$. In the Appendix the determination of $V$ is shown for Pattern 2.2. In Table 6 the $V$ values for all experimental patterns are given. Now, within the proposed model every interpretation, whether global or local, is treated equally with respect to the determination of its perceptual complexity. That is, both the complexity of the completed shape and the complexity of the amodal part of that shape are assumed to contribute to the perceptual complexity of an interpretation. We propose to quantify the perceptual complexity $(P)$ of an interpretation by a simple summation of those two complexities: $P=I+V$.

We hypothesize that $P$ is the lowest for the most preferred interpretation. To give an example, we apply this to the completions of Pattern 2.2. The perceptual complexities of the global and the local completions are $P_{\text {global }}=P(\mathrm{~A})=$ $I(\mathrm{~A})+V(\mathrm{~A})=9$ and $P_{\text {local }}=P(\mathrm{~B})=I(\mathrm{~B})+V(\mathrm{~B})=6$, respectively. Because $P(\mathrm{~B})<P(\mathrm{~A})$, Shape $\mathrm{B}$ is predicted to be preferred. If the perceptual complexity $(P)$ is used as a predictor for the data of Experiment 3, it appears that, for 10 out of 12 patterns, the most preferred interpretation bears the lowest perceptual complexity. The correlation coefficient of the proportion of preference for the global completion (Pref global local $)$ with the corresponding proportion of the perceptual complexities $P_{\text {globallocal }}=P_{\text {global }} /\left(P_{\text {global }}+\right.$ $\left.P_{\text {local }}\right)$ is $-.69(p<.01)$. Note that the proportion of the theoretical perceptual complexities expresses the strength of the global completion relative to the local completion.

To complete this analysis, the theoretical complexity of the amodal part is modified by extracting redundancy from the series of virtual elements by means of encoding, in the same way as has been done for the completed shapes. We refer to this measure with $V^{\prime}$. Notice that for every occluded shape, $V^{\prime}$ is always equal to, or smaller than, $V$. (In the Appendix, this is shown for Pattern 2.2, and in Table 6 the $V^{\prime}$ values for all experimental patterns are given.) Now, the perceptual complexity of each interpretation is taken to be $P^{\prime}=I+V^{\prime}$.

Applying this to the completions of Pattern 2.2, the values of the perceptual complexities are $P_{\text {global }}^{\prime}=P^{\prime}(\mathrm{A})=$ $I(\mathrm{~A})+V^{\prime}(\mathrm{A})=4+3=7$ and $P_{\text {local }}^{\prime}=P^{\prime}(\mathrm{B})=I(\mathrm{~B})+$ $V^{\prime}(\mathrm{B})=5+1=6$. Note that $P^{\prime}(\mathrm{A})$ is closer to $P^{\prime}(\mathrm{B})$ than $P(A)$ and $P(B)$ were in the previous proposal, but again Shape B is predicted to be preferred. Testing the perceptual complexity $P^{\prime}$ on the data reveals 11 out of 12 patterns for which the most preferred completion has the lowest perceptual complexity. The correlation coefficient of Pref $_{\text {globalllocal }}$ with the proportion of the perceptual complexities $P_{\text {globalllocal }}^{\prime}-.84(p<.001)$.

The above analysis shows that a combination of just two structurally describable factors already predicts the data

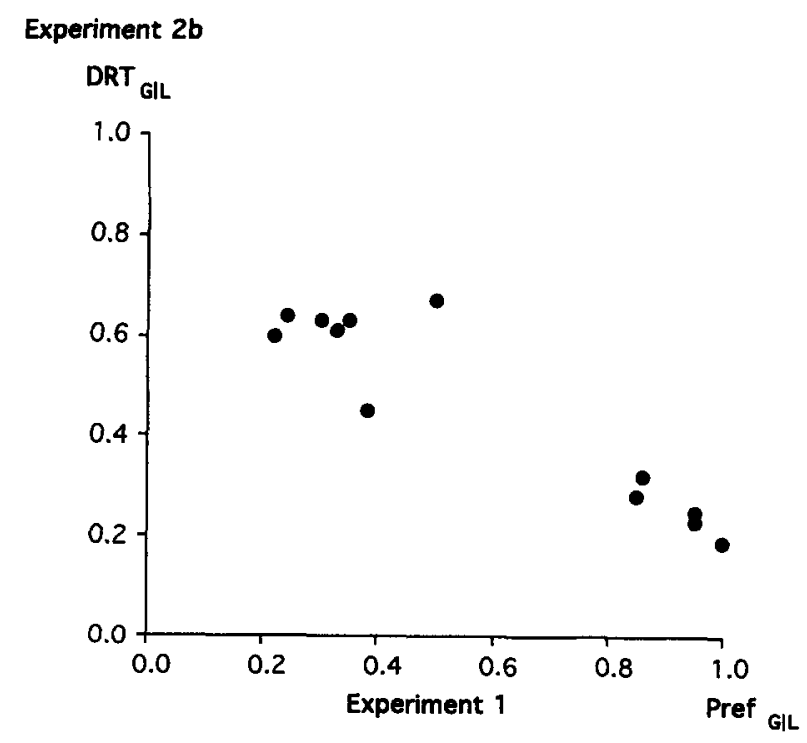

Figure 11. The results of Experiment $1\left(\operatorname{Pref}_{\mathrm{GIL}}\right)$ are plotted against the results of Experiment $2 \mathrm{~B}$. $\left(\mathrm{DRT}_{\mathrm{G} / \mathrm{L}}\right)$. Pearson's correlation coefficient is -.93 . Pref $=$ preference; $G=$ global; $L=$ local; DRT $=$ difference in response times. 
Table 6

Complexities for the Global and Local Completion of Each Global-Local-Divergent Pattern

\begin{tabular}{|c|c|c|c|c|c|c|}
\hline \multirow[b]{2}{*}{ Pattern } & \multicolumn{3}{|c|}{ Global } & \multicolumn{3}{|c|}{ Local } \\
\hline & $I$ & $V$ & $\overrightarrow{V^{\prime}}$ & $I$ & $V$ & $V^{\prime}$ \\
\hline 1.1 & 5 & 5 & 3 & 6 & 1 & 1 \\
\hline 1.2 & 4 & 3 & 3 & 8 & 1 & 1 \\
\hline 1.3 & 5 & 3 & 3 & 8 & 1 & 1 \\
\hline 1.4 & 5 & 5 & 3 & 8 & 1 & 1 \\
\hline 1.5 & 4 & 3 & 3 & 9 & 1 & 1 \\
\hline 1.6 & 5 & 3 & 3 & 9 & 1 & 1 \\
\hline 2.1 & 5 & 7 & 4 & 6 & 1 & 1 \\
\hline 2.2 & 4 & 5 & 3 & 5 & 1 & 1 \\
\hline 2.3 & 5 & 5 & 5 & 8 & 1 & 1 \\
\hline 2.4 & 5 & 13 & 5 & 6 & 1 & 1 \\
\hline 2.5 & 4 & 9 & 4 & 5 & 1 & 1 \\
\hline 2.6 & 5 & 9 & 6 & 8 & 1 & 1 \\
\hline
\end{tabular}

Note. The complexity of the completed shape is given by $I$. The complexity of the amodal part of that shape is given by $V$ and $V^{\prime}$, which represent two different quantifications.

reasonably well. Within the present model, the quantification of global and local aspects proceeds within the same quantifying scheme. The proposed model can easily be expanded with other factors that can be expressed in terms of structural information. Such a factor is the coincidence between contours of the shapes. Consider, for example, Figure 12. For both Patterns A and B the global completion results in the same regular octagon. The local completion is also the same for both patterns. Note, however, that for Pattern A, the corners of the octagon coincide with the contours of the occluding square. Clearly, in this pattern the tendency to a global completion appears to be weaker (and the tendency to a local completion stronger) than in Pattern B. This type of coincidence can be conceived as a regularity between the shapes and can be described structurally. We argued elsewhere (van Lier et al., 1994) that such coinci-

Table 7

Experiment 3 (Drawing Task): Replication of Experiment 1 but Controlling for Orientation and Context for each Global-Local-Divergent Pattern

\begin{tabular}{cc}
\hline Pattern & Pref $_{\text {globallocal }}$ \\
\hline 1.1 & 0.29 \\
1.2 & 0.90 \\
1.3 & 0.43 \\
1.4 & 1.00 \\
1.5 & 0.96 \\
1.6 & 0.90 \\
2.1 & 0.13 \\
2.2 & 0.17 \\
2.3 & 0.00 \\
2.4 & 0.39 \\
2.5 & 0.17 \\
2.6 & 0.10 \\
\hline
\end{tabular}

Note. For each pattern the preference (Pref) for the global completion relative to the local completion is given ( Pref $_{\text {global local }}$ ). Pref $_{\text {globallocal }}=$ number of global completions/(number of global completions + number of local completions).

dences increase the perceptual complexity of an interpretation. As mentioned already, in the present pattern such coincidences were avoided and therefore did not add to the perceptual complexity of global and local shapes as defined within the model.

The main characteristic of the presented model is that each interpretation is evaluated in the same way on both global and local aspects by means of a quantification of these structurally describable aspects. Other aspects, however, such as orientation of shapes, the relative orientation of edges, or familiarity, cannot be described structurally so far. Some of these aspects could in principle be implemented in a predictive model by assigning weighing factors to the perceptual complexities of different types of completions. However, the magnitudes of such weighing factors will have to be determined experimentally and will not be a consequence of our theory.

\section{General Discussion and Conclusion}

In concordance with various researchers (cf. Gregory \& Gombrich, 1973; Herbart, 1850; Leeuwenberg \& Buffart, 1983) we consider the selection of an interpretation as the result of a competition between various interpretations. The existence of a theoretical measure that provides a high correlation between the ratio of theoretical complexities of alternative interpretations and the ratio of the actual preferences supports such a competition.

One question that may arise is which completions are actually competing? It seems implausible that the perceptual system generates all possible completions. This would mean, for example, that Interpretation B in Figure 2 is also generated. The amount of different completions therefore will be innumerable. We do not think that the perceptual process actually generates all possible completions. In our view the generation of completions is constrained such that only "compatible extensions" of the visible structure are produced. We leave exact definitions of these compatible extensions for a forthcoming study but give here a rough sketch of the idea.

The pattern in Figure 13 can easily be interpreted as a

A

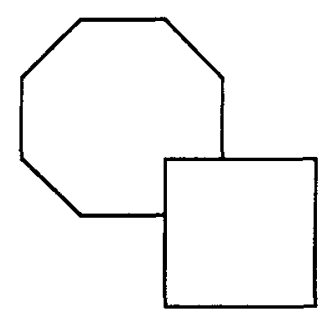

Figure 12. For both Patterns A and B the global completion results in the same regular octagon. The local completion is also the same for both patterns. In Pattern A the corners of the octagon coincide with the contours of the occluding square. In this pattern the tendency to a global completion appears to be weaker (and the tendency to a local completion stronger) than in Pattern B. 


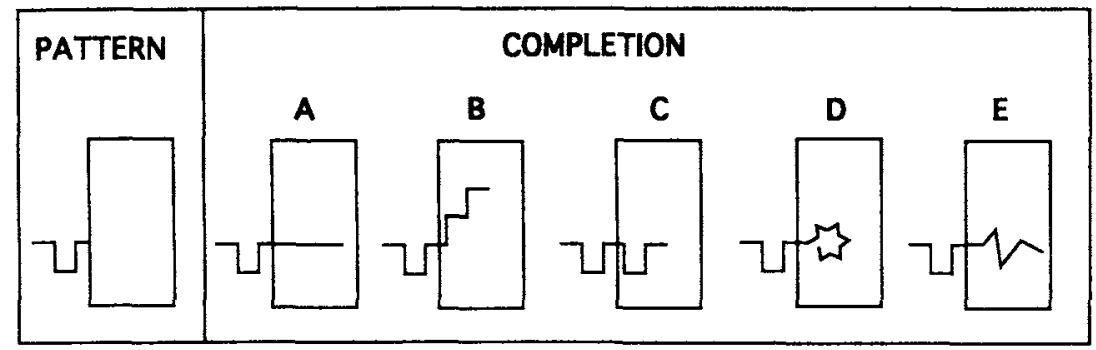

Figure 13. The pattern can easily be interpreted as a rectangle occluding a wirelike shape. Completions $\mathrm{A}, \mathrm{B}$, and $\mathrm{C}$ all represent compatible extensions of the visible structure, because they all repeat parts of the visible structure. Completions D and E cannot be conceived in that way.

rectangle occluding a wirelike shape. Roughly speaking, a compatible extension repeats parts of the visible structure. In Figure 13, Completion A repeats a small line segment of the line that disappears behind the occluding surface. Completion $B$ repeats a larger part of the visible structure, whereas Completion $\mathrm{C}$ repeats the whole visible structure. Completions $\mathrm{D}$ and $\mathrm{E}$ cannot be conceived as repetitions of visible parts. Therefore, they are not compatible extensions and should be excluded a priori. With respect to virtual angles, compatible extensions need a further specification: All angles formed by at least one virtual line should be repetitions of angles within the visible part. Consider again Figure 1. All virtual angles of the global completion (Shape A) are connected by at least one virtual line. In this case, each virtual angle indeed is a repetition of a visible angle. The local completion (Shape B in Figure 1) introduces an angle that cannot be conceived as a repetition of a visible angle. However, in this case the virtual angle results from a continuation. According to our definition of virtual lines, the virtual angle is not connected with virtual lines and therefore still is a compatible extension. We call the described restriction on extensions the compatibility of elements.

It is evident that a repetition of visible elements introduces additional identities between elements. Regularities, such as symmetries, iterations, and alternations, depend on the order of the elements. The regularities of the visible structure form the basis for a second constraint on compatible extensions, namely the compatibility of regularity. This means that only those interpretations are considered in which the completed shape bears the same kinds of regularity as the visible part does.

In future research the impact of both constraints on the completion process needs to be investigated. In our view such constraints will pave the way toward a concept of a generative process in pattern completion that yields a restricted number of interpretations comprising both global and local completions. This agrees with the presented model in which all interpretations are treated in the same way, so that the distinction between global and local completions appears to be post hoc and therefore irrelevant in the explanation of perceived pattern interpretations.

\section{References}

Attneave, F. (1982). Prägnanz and soap bubble systems: A theoretical exploration. In J. Beck (Ed.), Organization and representation in perception (pp. 11-29). Hillsdale, NJ: Erlbaum.

Barrow, H., \& Tenenbaum, J. (1981). Interpreting line drawings as three-dimensional surfaces. Artificial Intelligence, 17, 75-116.

Biederman, I. (1987). Recognition by components: A theory of human image understanding. Psychological Review, 94, 115147.

Boselie, F. (1988). Local versus global minima in visual pattern completion. Perception \& Psychophysics, 43, 431-445.

Boselie, F., \& Wouterlood, D. (1989). The minimum principle and visual pattern completion. Psychological Research, 51, 93-101.

Boselie, F., \& Wouterlood, D. (1992). A critical discussion of Kellman and Shipley's (1991) theory of occlusion phenomena. Psychological Research, 54, 278-285.

Buffart, H., Leeuwenberg, E., \& Restle, F. (1981). Coding theory of visual pattern completion. Journal of Experimental Psychology: Human Perception and Performance, 7, 241-274.

Chapanis, A., \& McCleary, R. (1953). Interposition as a cue for the perception of relative distance. The Journal of General Psychology, 48, 113-132.

Dinnerstein, D., \& Wertheimer, M. (1957). Some determinants of phenomenal overlapping. American Journal of Psychology, 70, 21-37.

Gerbino, W., \& Salmaso, D. (1987). The effect of amodal completion on visual matching. Acta Psychologica, 65, 25-46.

Gregory, R., \& Gombrich, E. (1973). Illusion in nature and art. London: Duckworth.

Hatfield, G., \& Epstein, W. (1985). The status of the minimum principle in the theoretical analysis of visual perception. Psychological Bulletin, 97, 155-186.

Herbart, J. (1850). Lehrbuch zur psychologie [Handbook of psychology]. Leipzig, Germany: Leopold Voss Verlag.

Hochberg, J., \& McAlister, E. (1953). A quantitative approach to figural "goodness." Journal of Experimental Psychology, 46, 361-364.

Kanizsa, G. (1985). Seeing and thinking. Acta Psychologica, 59, 23-33.

Kanizsa, G., \& Gerbino, W. (1982). Amodal completion: Seeing or thinking? In J. Beck (Ed.), Organization and representation in perception (pp. 167-190). Hillsdale, NJ: Erlbaum.

Kellman, P., \& Shipley, T. (1991). A theory of visual pattern interpolation in object perception. Cognitive Psychology, 23, 141-221. 
Koffka, K. (1935). Principles of Gestalt psychology. New York: Harcourt, Brace, \& World.

Köhler, W. (1947). Gestalt psychology. New York: Liveright.

Leeuwenberg, E. (1969). Quantitative specification of information in sequential patterns. Psychological Review, 76, 216-220.

Leeuwenberg, E. (1971). A perceptual coding language for visual and auditory patterns. American Journal of Psychology, 84, 307-349.

Leeuwenberg, E., \& Buffart, H. (1983). An outline of coding theory: A summary of related experiments. In H.-G. Geissler, H. Buffart, E. Leeuwenberg, \& V. Sarris (Eds.), Modern issues in perception (pp. 1-47). Amsterdam: North-Holland.

Leeuwenberg, E., Mens, L., \& Calis, G. (1985). Knowledge within perception: Masking caused by incompatible interpretation. Acta Psychologica, 59, 91-102.

Mens, L., \& Leeuwenberg, E. (1988). Hidden figures are ever present. Journal of Experimental Psychology: Human Perception and Performance, 14, 561-571.

Peterson, M., \& Hochberg, J. (1989). Necessary considerations for a theory of form perception: A theoretical reply to Boselie and Leeuwenberg (1986). Perception, 18, 105-119.

Ratoosh, P. (1949). On interposition as a cue for the perception of distance. Proceedings of the National Academy of Science, 35, 257-259.

Restle, F. (1982). Coding theory as an integration of gestalt psychology and information processing theory. In J. Beck (Ed.), Organization and representation in perception (pp. 31-56). Hillsdale, NJ: Erlbaum.

Rock, I. (1983). The logic of perception. Cambridge, MA: MIT Press.
Rock, I. (1985). Perception and knowledge. Acta Psychologica, $59,91-102$.

Sekuler, A., \& Palmer, S. (1992). Perception of partly occluded objects: A microgenetic analysis. Journal of Experimental Psychology: General, 121, 95-111.

Shipley, T., \& Kellman, P. (1992). Perception of partly occluded objects and illusory figures: Evidence for an identity hypothesis. Journal of Experimental Psychology: Human Perception and Performance, 18, 106-120.

van der Helm, P. (1988). Accessibility and simplicity of visual structures. Unpublished doctoral dissertation, University of Nijmegen, Nijmegen, The Netherlands.

van der Helm, P., \& Leeuwenberg, E. (1991). Accessibility, a criterion for regularity and hierarchy in visual pattern codes. Journal of Mathematical Psychology, 35, 151-213.

van der Helm, P., van Lier, R., \& Leeuwenberg, E. (1992). Serial pattern complexity: Irregularity and hierarchy. Perception, 21, 517-544.

Von Helmholtz, H. (1962). Treatise on physiological optics, I-III (J. Southall, Trans.). New York: Dover. (Original work published 1867).

van Lier, R., van der Helm, P., \& Leeuwenberg, E. (1994). Integrating global and local aspects of visual occlusion. Perception, 23, 883-903.

Wertheimer, M. (1923). Untersuchungen zur Lehre von der Gestalt [Psychological research]. Psychologische Forschung, 4, 301350.

Wouterlood, D., \& Boselie, F. (1992). A good continuation model of some occlusion phenomena. Psychological Research, 54, 267-277.

\section{Appendix}

\section{Quantifying Complexities}

\section{Coding Rules and Information Load}

In structural information theory three types of regularity play a crucial role: iteration, symmetry, and alternation. The coding rules that describe these regularities are illustrated in Table A1. The encoding procedure starts with a symbol series and extracts as much regularity as possible from this symbol series. The result is a so-called minimum code. The number of parameters at all hierarchical levels in that minimum code is taken as the information load $I$ of that code, reflecting the amount of irregularity in the series (see van der Helm, van Lier, \& Leeuwenberg, 1992). For example, the encoding of "abab" results in " $2 \times(a b)$." The information load of this code is 3 , because of the symbols " $a$ " and " $b$ "

Table A1

The Three Types of Regularity Used in the Structural Information Theory

\begin{tabular}{lllc}
\hline Regularity & $\begin{array}{c}\text { Symbol } \\
\text { series }\end{array}$ & \multicolumn{1}{c}{ Code } & $\begin{array}{c}\text { Information } \\
\text { load }\end{array}$ \\
\hline Iteration & aa & $2 \times(\mathrm{a})$ & 1 \\
Symmetry & abcba & $\mathbf{S}[(\mathrm{a})(\mathrm{b}),(\mathrm{c})]$ & 3 \\
Alternation & abac & $\langle(\mathrm{a})\rangle /<(\mathrm{b})(\mathrm{c})\rangle$ & 3 \\
\hline
\end{tabular}

Note. Encoding of a symbol proceeds by extracting these regularities from the series. The information load of the code reflects the amount of irregularity in the series. (parameters at the lowest level) and the group "ab" (parameter at the highest level). The encoding procedure, from a symbol series to its minimum code, has been implemented in a computer algorithm called PISA (van der Helm, 1988).

\section{Determination of $I$ for a Shape}

In Figure A1, Pattern 2.2 and its global and local completions are given once more. The determination of the complexity of a shape starts with labeling all lines and angles such that all equal lines or angles are labeled with the same symbol. Next, the contour of each shape is represented by a symbol series in which the order of the contour elements is preserved. The encoding of the global shape results in the minimum code " $3 \times(\mathrm{S}[(k),(b)] a)$," which has an information load of 4 (parameters " $k$," " $b$," " $a$," and "(S $[(k),(b)] a)$." Therefore, $I(\mathrm{~A})=4$. The encoding of the local

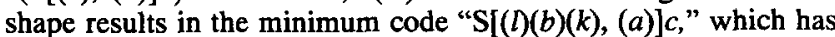
an information load of 5 (parameters " $l$," " $b$," " $k$," " $a$," and " $c$ "). Therefore, $l(B)=5$.

\section{Determination of $V$ and $V^{\prime}$}

In Figure A2, Pattern 2.2 and its global and local completions are given once more. $V$ is defined by the number of virtual elements. This number is given by the difference between the total number of contour elements of a shape and the total number of completely or partly visible contour elements of that shape. Con- 


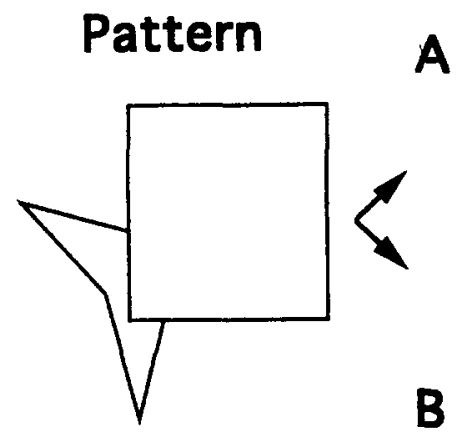

$\begin{array}{ll}\text { Primitive Code: } & \text { kbkakbkakbka } \\ \text { Minimal Code: } & 3^{\star}(S[(k),(b)] a) \\ l=4 & \end{array}$

$\begin{array}{ll}\text { Primitive Code: } & \text { Ibkakblc } \\ \text { Minimal Code: } & S[(l)(b)(k),(a)] c \\ I=5\end{array}$

Figure A1. The encoding procedure as performed for a two-dimensional shape. First, the contour of a shape is represented by a symbol series, such that equal lines or angles are represented by the same symbol and such that their order and identity are preserved. Second, the complexity of the shape is determined by the information load $(I)$ of the minimum code of that symbol series.

sequently, the continuation of a visible edge behind an occluding surface does not increase the complexity of the amodal part.

The global completion (A) of the pattern in Figure A2 requires five virtual elements, labeled " $a$," " $k$," " $b$," " $k$," and " $a$." Therefore, $V(A)=5$. The local competition (B) requires one virtual element, labeled " $c$." Therefore, $V(B)=1$.
The determination of $V^{\prime}$ proceeds by encoding the series of virtual elements. For the global completion the encoding of the

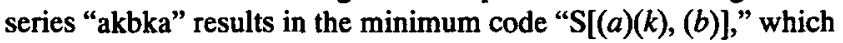
has an information load of 3 (parameters " $a$," " $k$," and " $b$ "). Therefore, $V^{\prime}(\mathrm{A})=3$. For the local completion the encoding obviously results in the symbol "c." Therefore, $V^{\prime}(B)=1$.

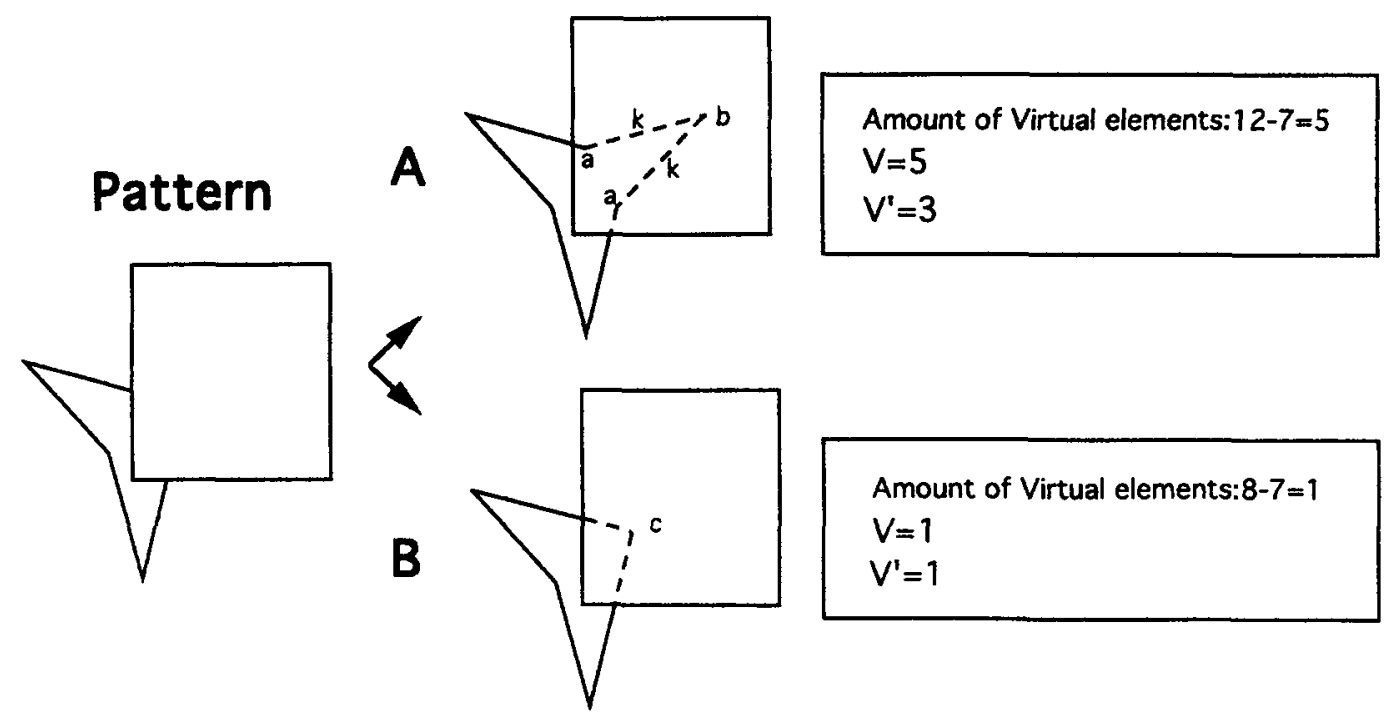

Figure A2. The complexity ( $V$ ) of the amodal part of a shape is given by the difference between the total number of contour elements of the shape and the total number of completely or partly visible contour elements of that shape. The complexity $\left(V^{\prime}\right)$ is obtained by encoding the series of virtual elements.

Received May 28, 1993 